\section{Infection with CagA-positive Helicobacter pylori strain containing three EPIYA C phosphorylation sites is associated with more severe gastric lesions in experimentally infected Mongolian gerbils (Meriones unguiculatus)}

\author{
M. Ferreira Júnior, ${ }^{1}$ S.A. Batista, ${ }^{2}$ \\ P.V.T Vidigal, ${ }^{3}$ A.A.C. Cordeiro, \\ F.M.S. Oliveira, ${ }^{1}$ L.O. Prata, ${ }^{1}$ \\ A.E.T. Diniz, ${ }^{4}$ C.M. Barral, ${ }^{4}$ \\ R.C. Barbuto, ${ }^{4}$ A.D. Gomes, ${ }^{2}$ \\ I.D. Araújo, ${ }^{4}$ D.M.M. Queiroz, ${ }^{2 *}$ \\ M.V. Caliari ${ }^{1 *}$ \\ 'Department of General Pathology, \\ Institute of Biological Sciences, \\ Federal University of Minas Gerais, \\ Belo Horizonte
}

'Laboratory of Research in Bacteriology, Faculty of Medicine, Federal University of Minas Gerais, Belo Horizonte ${ }^{3}$ Department of Anatomical Pathology, Faculty of Medicine, Federal University of Minas Gerais, Belo Horizonte ${ }^{4}$ Surgery Department, Faculty of Medicine, Federal University of Minas Gerais, Belo Horizonte, MG, Brazil

*Joint senior authors

\section{Abstract}

Infection with Helicobacter pylori strains containing high number of EPIYA-C phosphorylation sites in the CagA is associated with significant gastritis and increased risk of developing pre-malignant gastric lesions and gastric carcinoma. However, these findings have not been reproduced in animal models yet. Therefore, we investigated the effect on the gastric mucosa of Mongolian gerbil (Meriones unguiculatus) infected with CagApositive $H$. pylori strains exhibiting one or three EPIYA-C phosphorilation sites. Mongolian gerbils were inoculated with $H$. pylori clonal isolates containing one or three EPIYA-C phosphorylation sites. Control group was composed by uninfected animals challenged with Brucella broth alone. Gastric fragments were evaluated by the modified Sydney System and digital morphometry. Clonal relatedness between the isolates was considered by the identical RAPD-PCR profiles and sequencing of five housekeeping genes, vac $\mathrm{A} \mathrm{i} / \mathrm{d}$ region and of oipA. The other virulence markers were present in both isolates (vacA slild1m1, ice $\mathrm{A} 2$, and intact $\operatorname{dupA}$ ). CagA of both isolates was translocated and phosphorylated in AGS cells. After 45 days of infection, there was a significant increase in the number of inflammatory cells and in the area of the lamina propria in the infected animals, notably in those infected by the CagA-positive strain with three EPIYA-C phosphorylation sites. After six months of infection, a high number of EPIYA-C phosphorylation sites was associated with progressive increase in the intensity of gastritis and in the area of the lamina propria. Atrophy, intestinal metaplasia, and dysplasia were also observed more frequently in animals infected with the CagA-positive isolate with three EPIYA-C sites. We conclude that infection with $H$. pylori strain carrying a high number of CagA EPIYA-C phosphorylation sites is associated with more severe gastric lesions in an animal model of $H$. pylori infection.

\section{Introduction}

Helicobacter pylori is a Gram-negative spiral bacterium that chronically infects the gastric mucosa of more than $50 \%$ of the world population. ${ }^{1}$ The infection is associated with chronic gastritis, gastric and duodenal peptic ulcer, gastric adenocarcinoma and gastric mucosaassociated lymphoid tissue (MALT) lymphoma. ${ }^{2}$ Inflammation, atrophy, intestinal metaplasia, dysplasia, and gastric carcinoma are noteworthy ${ }^{3}$ among the histological changes in the gastric mucosa caused by the colonisation of $\mathrm{H}$ pylori.

It is well demonstrated that there is a geographic variation among the $H$. pylori strains that exhibit a broad range of genetic diversity. A striking difference that characterises more virulent strains is the presence of the cag PAI (cag pathogenicity island) with approximately 40-Kb consisting of 27-31 genes. ${ }^{4}$ Among them, the cagA gene encodes the CagA protein that possesses carcinogenic properties. The island is responsible for coding a type IV secretion system (T4SS), which allows the entrance of CagA protein inside the gastric epithelial cells, followed by protein phosphorylation and the activation of proinflammatory and antiapoptotic genes that increase the risk of developing precancerous lesions. ${ }^{5,6}$ CagA phosphorylation occurs on tyrosine residues in a repeated fiveamino-acid sequences located in the carboxyl terminus, denominated EPIYA (Glu, Pro, Ile, Tyr and Ala) A, B, C, and D according to different flanking amino acids. The D (Eastern) and C (Western) are the main sites for CagA phosphorylation by multiple members of the Src kinase family. Phosphorylated CagA forms a
Correspondence: Prof. Marcelo Vidigal Caliari, Department of General Pathology, Institute of Biological Sciences, Federal University of Minas Gerais, Av. Antônio Carlos 6627, Belo Horizonte, MG, CEP 31 270-010, Brazil.

Tel. +55.31.34092892 -Fax: +55.31.34092879.

E-mail: caliari@icb.ufmg.br

Key words: Gastritis, atrophy, intestinal metaplasia, dysplasia, Mongolian gerbil, cagA EPIYA C motif.

Contributions: SAB, ADG, DMMQ, DNA extraction, RUT, analysis of CagA translocation and DNA sequencing; PVTV, IDA, classification and grading of gastritis by the Sydney system and macroscopic analysis of the gastric mucosa; MFJ, MVC, AETD, CMB, RCB, inoculation of the animals, necropsy and histopathological techniques; MFJ, FMSO, AACC, LOP, immunohistochemical techniques; MVC, AACC, MFJ, morphometric analysis of the inflammatory infiltrate and the area of the antral lamina propria; DMMQ, Data analysis; DMMQ, MVC, MFJ, wrote the paper; DMMQ, MVC, IDA ,designed the study, supervised the laboratory works. All authors read and approved the manuscript.

Conflict of interest: the authors declare no conflict of interest.

Funding: this work was financially supported by the Dean of Research of the Federal University of Minas Gerais (Pró-Reitoria de Pesquisa da Universidade Federal de Minas GeraisPRPq/UFMG), The Minas Gerais State Research Foundation (Fundação de Amparo à Pesquisa do Estado de Minas Gerais - FAPEMIG), and the National Council for Research and Development (Conselho Nacional de Pesquisa $e$ Desenvolvimento - CNPq).

Received for publication: 4 February 2015. Accepted for publication: 4 April 2015.

This work is licensed under a Creative Commons Attribution NonCommercial 3.0 License (CC BYNC 3.0).

(C) Copyright M. Ferreira Júnior et al., 2015 Licensee PAGEPress, Italy

European Journal of Histochemistry 2015; 59:2489 doi:10.4081/ejh.2015.2489

complex with SHP-2 phosphatase triggering abnormal cellular signals that lead to deregulation of cell growth, cell to cell contact and cell migration, elongation of epithelial cells and increase of epithelial cell turnover, which enhance the risk of acquiring precancerous changes. ${ }^{5,7}$ Human infection with CagA-positive $H$. pylori strains containing high number of EPIYA-C phosphorylation sites is associated with a more severe chronic gastritis and an increased risk of developing intestinal metaplasia and gastric cancer. ${ }^{6,8}$ However, we are 
unaware of studies confirming, in animal models, the associations observed in human beings.

The aim of this study was therefore to evaluate qualitatively and through digital morphometry, the histological alterations induced by the infection with CagA-positive $H$. pylori strains with different numbers of EPIYA-C on the gastric mucosa of Mongolian gerbil.

\section{Materials and Methods}

\section{H. pylori strains}

Mongolian gerbil is more easily colonized by $H$. pylori strain that was recently isolated, without many subcultures. Thus, fragments of the antral mucosa of the stomach obtained at surgery from a 71-year-old patient with gastric carcinoma was smeared on BHM agar plates and incubated in microaerophilic conditions at $37^{\circ} \mathrm{C} .^{9}$ After $48 \mathrm{~h}$ of growth, the entire population of bacterial cells recovered from the plates was harvested and stored at $-70^{\circ} \mathrm{C}(H$. pylori stock collection from the Laboratory of Research in Bacteriology, Faculty of Medicine, Federal University of Minas Gerais). Singlecolonies of $H$. pylori, obtained from the original culture, were propagated as pools for less than 10 passages before freezing at $-70^{\circ} \mathrm{C}$. The growth of each colony was tested for EPIYA-C patterns by PCR according to Yamaoka et al. ${ }^{10}$ and the results were confirmed by sequencing. ${ }^{6}$ Two isolates, LPB 1034-14 and LPB 1034-6, containing one or three EPIYA-C phosphorylation sites, were used to inoculate the animals. Strains isolated from adifferent individuals show substantial genomic diversity, although isolates from single individual or family members are frequently clonal. ${ }^{11}$ Clonal relatedness between the isolates was evaluated by Random-amplified DNA (RAPD)-PCR by using two sets of primers according to Akopyanz et al. ${ }^{12}$ and sequencing of the house- keeping genes efp (564 kb), yphC (724 kb), atpA $(829 \mathrm{~kb})$, ureI $(670 \mathrm{~kb})$ and mut $\mathrm{Y}(652$ $\mathrm{kb})^{13}$ and the virulence genes involved in the inflammatory response to the infection; vacA i/d regions, CT dinucleotide repeated motif region of oip $\mathrm{A}^{14,15}$ and intact dupA. ${ }^{16}$ The presence of other cag PAI genes involved in the translocation of the cagA to the host cells (cagl, $\operatorname{cag} \mathrm{L}, \operatorname{cagX}, \operatorname{cag} \mathrm{Y}$ and $\operatorname{cag} \mathrm{T}$ ) and $\operatorname{cag} \mathrm{E}^{17}$ and the genotype of vacA and ice $\mathrm{A}^{18}$ were also evaluated. All primers and PCR conditions are listed in Supplementary Table 1. Two days culture of the isolates was prepared for the inocula. The cells were harvested in Brucella broth (difco). The concentration was adjusted according to $\mathrm{OD}$ at $550 \mathrm{~nm}$ and the cells were administered to the animals immediately after harvesting.

\section{Analysis of CagA translocation}

CagA translocation was confirmed by Western blotting and immunoprecipitation as proposed by Liang et al. ${ }^{19} \mathrm{Cag}$ PAI functionality was evaluated by investigating the translocation followed by phosphorylation of the CagA EPIYA-C from the isolates into the human gastric epithelial AGS cells (ATCC ${ }^{\circledR}$ CRL1739 $^{\text {TM }}$ ). The cells were cultured in RPMI 1640 medium (Invitrogen, São Paulo, Brazil), containing $10 \%$ FBS (Invitrogen), incubated at $37^{\circ} \mathrm{C}$ in a fully humidified atmosphere with $5 \% \mathrm{CO}_{2}$ in air. On the day of the experiment, confluent cells were incubated overnight in fresh serum and antibiotic-free media. AGS cells were coincubated with $H$. pylori up to multiplicities of infection of 100:1. After six hours the cells were washed four times with PBS and lysed with a lysis buffer containing $50 \mathrm{mM}$ Tris $(\mathrm{pH}$ 7.5), 5 mM EDTA, $100 \mathrm{mM} \mathrm{NaCl}$, 1\% Triton X100, $1 \mathrm{mM}$ phenylmethylsulfonyl fluoride, and protease inhibitors (Roche Applied Science). To confirm the CagA translocation, proteins in the whole cell extracts $(30 \mu \mathrm{g})$ were separated by SDS-PAGE (4-12\% gels) and electrotransferred to nitrocellulose membranes (Invitrogen). After, blotting was blocked in a solution of $3 \%$ skim milk, $0.05 \%$ Tween 20 , and TBS, the membrane-bound proteins were probed with primary monoclonal antibody against CagA (A-10, Santa Cruz Biotechnology, Santa Cruz, CA, USA). The membranes were washed and then incubated with horseradish peroxidase-conjugated secondary antibody for $60 \mathrm{~min}$. Antibody-bound protein bands were detected using enhanced chemiluminescence reagents (Millipore, Billerica, MA, USA) and captured with ImageQuant 350 (GE Healthcare, Uppsala, Sweden).

Immunoprecipitation was performed using $1 \mathrm{mg}$ of the lysate protein obtained from the AGS cells co-incubated with the $H$. pylori isolates. Each sample was incubated overnight at $4^{\circ} \mathrm{C}$ with monoclonal anti-H.pylori CagA antibody (A-10, Santa Cruz Biotechnology) followed by four hours at $4^{\circ} \mathrm{C}$ with $30 \mu \mathrm{L}$ aliquot of protein G Sepharose beads (Invitrogen). The beads were washed five times with cold lysis buffer, and proteins were eluted by boiling for $10 \mathrm{~min}$ in $2 \mathrm{x}$ electrophoresis sample buffer containing $5 \mathrm{mmol} / \mathrm{L}$ Tris, $10 \%$ sodium dodecyl sulfate, 12\% 2-mercaptoethanol, 20\% glycine, and 1\% Bromophenol blue. Immunoprecipitated proteins were subjected to SDS-PAGE, transferred to nitrocellulose membranes and incubated with monoclonal anti p-tyr antibody (pY-99, Santa Cruz Biotechnology). The bands were identified by using a chemiluminescence reagent (Millipore) and captured with ImageQuant 350 (GE Healthcare).

\section{DNA extraction}

DNA was extracted with the QIAamp kit (QIAGEN, Hilden, Germany) according to the manufacturer's instructions with slight modifications. ${ }^{20}$ The DNA concentration was determined by spectrophotometry using the Ultrospec $^{\circledR} 2100$ Pro apparatus (Amersham Biosciences, Uppsala, Sweden), and the extracted DNA was kept at $-20^{\circ} \mathrm{C}$ until use.

Table 1. Gastric inflammation, inflammatory activity and the number of lymphoid follicles in Mongolian gerbils infected with Helicobacter pylori 45 days and six months post-inoculation.

\begin{tabular}{|c|c|c|c|c|c|c|c|c|c|}
\hline \multirow[t]{2}{*}{ Groups } & \multicolumn{4}{|c|}{ Inflammation (MN cells) } & \multicolumn{3}{|c|}{ Activity (PMN cells) } & \multirow[b]{2}{*}{+++} & \multirow[t]{2}{*}{ LF } \\
\hline & 0 & + & ++ & +++ & 0 & + & ++ & & \\
\hline \multicolumn{10}{|l|}{45 days } \\
\hline CTRL & 8 & 0 & 0 & 0 & 8 & 0 & 0 & 0 & 0 \\
\hline CagAlEp & 0 & 6 & 2 & 0 & 2 & 6 & 0 & 0 & 3 \\
\hline CagA3Ep & 0 & 3 & 5 & 0 & 0 & 5 & 3 & 0 & 12 \\
\hline \multicolumn{10}{|l|}{ Six months } \\
\hline CTRL & 8 & 0 & 0 & 0 & 8 & 0 & 0 & 0 & 0 \\
\hline CagAlEp & 0 & 2 & 6 & 0 & 1 & 4 & 3 & 0 & 13 \\
\hline CagA3Ep & 0 & 1 & 4 & 3 & 0 & 2 & 3 & 3 & 15 \\
\hline
\end{tabular}

MN, mononuclear cells; PMN, polymorphonuclear cells; LF, lymphoid follicles; CTRL, control group consisting of uninfected animal; CagAlEp, CagA-positive with one EPIYA-C group; CagA3Ep, CagA-positive with three EPIYA-C group; 0 , absent; +, mild; ++ moderate; +++ , severe. Significant differences were observed when the controls were compared with the CagA3Ep and with the CagAlEp animals regarding the scores of MN $(\mathrm{P}<0.001)$ and of PMN $(\mathrm{P} \leq 0.003)$ cells after 45 days and six months of infection. Data were analysed by the two tailed Mann-Whitney $\mathrm{U}$ test. 


\section{H. pylori specific urea, cagA and cagA 3' variable region in the gastric mucosa}

The primers and conditions used for the amplification of urea and cagA were previously described by Clayton et al. ${ }^{21}$ and Peek et al. ${ }^{22}$ and the cagA 3' region was amplified according to Yamaoka et al..$^{10}$ (Supplementary Table 1).

Sequencing of the cagA 3' variable region, vacA $\mathrm{i} / \mathrm{d}$ region, intact dupA, and CT dinucleoptide repeat motif region of oipA

In general, PCR products were purified with the Wizard SV Gel and PCR Clean-Up System (Promega, Madison, MI, USA) according the manufacturer's recommendations and sequenced using an ABI Prism BigDye Terminator Cycle Sequencing kit in an ABI Prism 310 automatic sequencer (Applied Biosystems, Foster City, CA, USA). The sequences obtained were aligned using the CAP3 Sequence Assembly Program (http:// pbil.univ-lyon1.fr/cap3.php). Regarding the dupA, the primer walking methodology using five primer sets was employed (Supplementary Table 1). ${ }^{16}$ After alignment, nucleotide sequences of the cagA 3' variable region and $v a c \mathrm{~A} \mathrm{i} / \mathrm{d}$ region were transformed into aminoacid sequences using the Blastx program (http://blast.ncbi.nlm.nih. gov/ Blast.cgi). The nucleotide sequences of the gene loci of the gene strains were deposited in GenBank under the following accession numbers: efp GenBank accession numbers KP723366, KP723367, KP723368; $y p h C$ GenBank accession numbers KP723369, KP723370, KP723371; atpA GenBank accession numbers KP723372, KP723373, KP723374; ureI GenBank accession numbers KP723375, KP723376, KP723377; mutY GenBank accession numbers KP723378, KP723379, KP723380.

\section{Animals}

Forty-eight three-month-old female Mongolian gerbils obtained from the animal house of the Institute of Biological Sciences/Universidade Federal de Minas Gerais were maintained with appropriate nutrition, ventilation and lighting conditions and handled in accordance with the ethical standards of the UFMG. They were divided into three experimental groups: a control group consisting of uninfected animals challenged with Brucella broth alone (CTRL), animals inoculated with $H$. pylori CagA-positive strain with one EPIYA-C segment (CagA1Ep), and animals inoculated with $H$. pylori CagApositive strain with three EPIYA-C segments (CagA3Ep). Each group consisted of 16 animals; eight animals from each group were sacrificed 45 days after the inoculation, and the remaining eight animals were sacrificed six months after the infection.

Prior to the inoculation, the animals were fasted for eight hours and slightly sedated with isoflurane. The inocula of $0.8 \mathrm{~mL}$ of the bacterial suspensions containing $10^{9} \mathrm{CFU} / \mathrm{mL}$ in sterile Brucella broth or Brucella broth alone (control group) were administered by intragastric gavage three times at intervals of $48 \mathrm{~h}$. Four h after the inoculation, the animals received standard diet for rats (Labina ${ }^{\circledR}$, Purina, Brazil) and water ad libitum.

\section{Rapid urease test}

The rapid urease test (RUT) was performed by incubating one gastric antral fragment into a semi-solid agar containing phenol red $2 \%$ and urea $0.01 \mathrm{M}$ at $37^{\circ} \mathrm{C}$ for $24 \mathrm{~h}$.

\section{Histopathological analysis and semi-quantitative evaluation of spiral bacterium}

After abdominal incision, the stomach was removed and opened along the greater curvature. Mucosa fragments were obtained along the lesser and greater curvatures, including the regions of the gastric body and gastric antrum for DNA, RUT and histology. For histological processing, the fragments were fixed in $10 \%$ buffered formalin for $48 \mathrm{~h}$. Four $\mu \mathrm{m}$ thick tissue sections were stained with Hematoxylin and Eosin (H\&E) for histological analyses and Silver staining confirmed by Carbolfuchsin staining for the semi quantitative evaluation of the spiral bacteria load in scores (from 0 to 3 ) in the gastric glands. ${ }^{23}$ Single organism or no more than two small clusters of stainable organisms were scored as 1 , three to six clusters were scored as 2 and more than six as $3 .^{24}$ The H\&E stained sections were used for the histopathological analysis and the classification of gastritis using the Sydney system, ${ }^{3}$ as well as for the morphometric analysis of the inflammatory infiltrate and calculation of the antral lamina propria area. The presence of intestinal metaplasia was confirmed by using the PAS-Alcian Blue technique, $\mathrm{pH} 2.5$, which stains acid mucins.

\section{Classification and grading of the gastritis by the modified Sydney system}

The degree of gastric inflammation (mononuclear cells; MN) and activity (polymorphonuclear cells; PMN) was evaluated by the modified Sydney system ${ }^{3}$ and classified as follows: absent (no inflammatory cells), mild, moderate, and severe, according to the number of inflammatory cells. The presence of atrophy, intestinal metaplasia, and dysplasia as well as lymphoid follicles were also investigated.

\section{Morphometric analysis of the inflammatory infiltrate and antral lamina propria area}

All histological sections were analysed with a 40x objective, and 15 images of the gastric antral mucosa were captured using a Q-Color3 microcamera (Olympus, Tokyo, Japan). A total area of $1.37 \times 10^{6} \mu \mathrm{m}^{2}$ of mucosa was analysed for each case. Algorithms from the KS300 software (Carl Zeiss, Oberkochen, Germany) were used for image processing and segmentation, definition of the morphometric conditions, cell counting, and calculation of the area of the lamina propria. Segmentation allowed to select the nuclei of all cell types present in the lamina propria of the antral mucosa and to exclude the cell cytoplasm and other structures present in the histological sections. By this process, a binary image was created, and the nuclei of all cell types presented in the antral mucosa were counted. The result obtained in the control animals was considered the expected cellular pattern of the antral mucosa without inflammatory infiltration. In the infected animals, the nuclei of the cell types normally present in the lamina propria of the antral mucosa and nuclei of leukocytes recruited in the inflammatory process were counted, allowing a quantitative assessment of inflammation by a method that we previously used in other studies..$^{25}$ Subsequently, all pixels of the lamina propria were selected to obtain another binary image to calculate the area in $\mu \mathrm{m}^{2}$.

\section{Statistical analysis}

The comparison between the degree of inflammation and activity of the gastritis as well as the scores of the spiral microorganism among the groups were evaluated by the two tailed Mann-Whitney U test. Because the number of inflammatory cells and the area of the antral lamina propria were not normally distributed, even after log transformation, the morphometric data were compared among the different groups and periods of infection by the two tailed Mann-Whitney $\mathrm{U}$ test. P values $\leq 0.05$ were considered significant.

\section{Results}

\section{Comparison of the two H. pylori isolates}

The isolates yielded cagA PCR products with different size of 640 and 850 bp (Figure 1a). The sequence analyses demonstrated an insertion of 204-bp in the isolate LPB1034-6 compared with the isolate LPB1034-14. The cagA 
sequences were $99.2 \%$ identical (Figure 1c). Translation of the nucleotide sequences identified an insertion that corresponds to two identical EPYIA-C motifs and multimerization sites in the LPB1034-6 isolate (Figure 1d).

The strains were considered closely related, by the identical RAPD-PCR profiles (Figure 1b) and identical oipA, with 9 CT (Supplementary Figure 1a), vacA i/d (Supplementary Figure 1b), and five housekeeping genes sequences (efp GenBank accession number 1794575; yphC GenBank accession number 1794676; atpA GenBank accession number 1794697; ureI GenBank accession number 1794705; mutY GenBank accession number 1796141). Since the $c a g \mathrm{E}, \operatorname{cagl}, \operatorname{cag} \mathrm{L}, \operatorname{cagX}, \operatorname{cag} Y$ and $\operatorname{cag} \mathrm{T}$ genes are involved in the induction of IL-8 secretion and the lack of one of them may impair the CagA translocation, their presence was also evaluated and confirmed in both isolates. Furthermore, the genotypes of the other bacterium virulence markers involved in gastric inflammation (vacA slild1m1, iceA2, and intact $d u p \mathrm{~A}$ with $1884 \mathrm{bp}$ ) were identical in both isolates.

\section{Translocation of CagA into AGS cell}

The lysates obtained after co-incubation of the isolates and AGS cells were subjected to immunoprecipitation and Western blot using anti-CagA and anti-pY99 monoclonal antibodies. The results in Figure 2 show that the precipitate protein reacted with both anti-CagA and anti-phosphotyrosine demonstrating that CagA is the tyrosine phosphorylated protein.

\section{H. pylori status}

The animals were considered $H$. pylori infected when two were positive among the tests. RUT was positive in $84.4 \%$ of the infected animals and negative in all control animals. The ure A as well cagA genes were present in the gastric mucosa of all infected animals, but not in the gastric mucosa of the control animals. PCR to detect the number of CagA EPIYA$\mathrm{C}$ motifs was positive in 45 days and six months post-infection in all infected animals. The EPIYA-C PCR results were confirmed by sequencing without differences before and after inoculation (Figure $1 \mathrm{c}, \mathrm{d}$ ). The virulence genes remained present as detected by PCR.

In the inoculated animals, the degree of stainable spiral organism varied from 1 to 3 (median=2) in both groups infected with strains containing one or three EPIYA-C segments either 45 days or six months post-infection without differences between the groups $(\mathrm{P}=0.53)$. The bacterial load was higher in the antral (median=2) than in the oxyntic (median=1) gastric mucosa in both infected animal groups $(\mathrm{P}=0.04)$.

\section{Macroscopic analysis of the gastric mucosa}

Forty-five days after the infection, none of the control animals exhibited macroscopic alterations in the gastric mucosa. Six months post-infection, macroscopic lesion continued to be absent in the control group (Figure 3a). Among the eight animals from the CagAlEp group, one exhibited a non-ulcerative flat red/purple haemorrhagic lesion in the oxyntic mucosa (Figure $3 b)$. A reduction of the oxyntic mucosa as a consequence of an expansion of the antral mucosa area was observed in the stomach of another animal from the CagAlEp group and of four animals from the CagA3Ep group. In one animal from the CagA3Ep group, the expansion of the antral mucosa was more
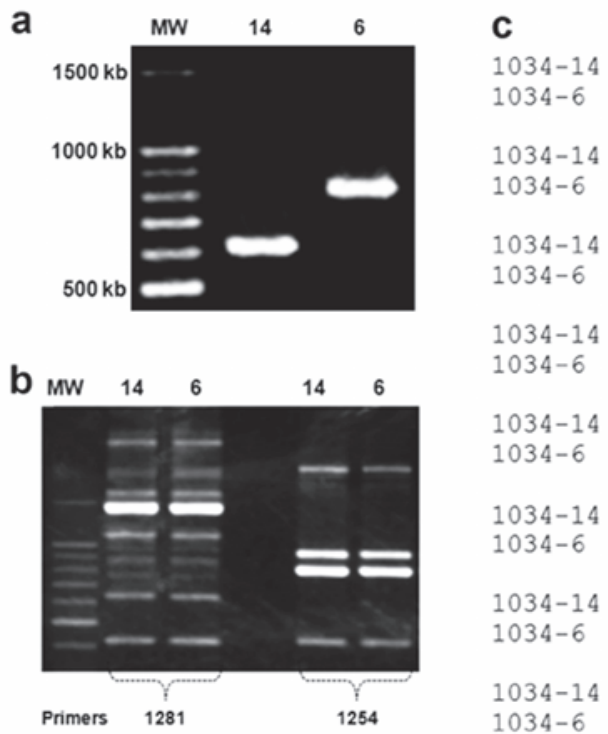

GAACCCATTTATGCTAAAGTTAATAAAAAGAAAACAGGACAAGTAGCTAGCCCTGAAGA - 59 GAACCCATTTATGCTAAAGTTAATAAAAAGAAAACAGGACAAGTAGCTAGCCCTGAAGA - 59

ACCCATTTAT GCTCAAGTTGCTAAAAAGGTAAATGCAAAAATTGACCGACTCAATCAAA - 118 ACCCATTTATACTCAAGTTGCTAAAAAGGTAAATGCAAAAATTGACCGACTCAATCAAA - 118

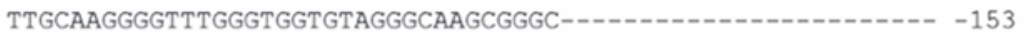
TTGCAAGGGGTTTGGGTGGTGTAGGGCAAGCGGGCTTCCCTTTGAAAAGGCATGATAAA - 177

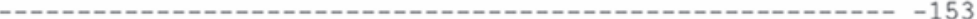
GTTGATGATCTCAGTAAGGTAGGGCGATCAGTTAGCCCTGAACCCATTTATGCTACAAT - 236

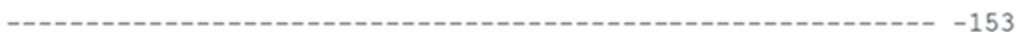
TGATGATCTCGGCGGACCTTTCCCTTTGAAAAGGCATGATAAAGTTGATGATCTCAGTA - 295

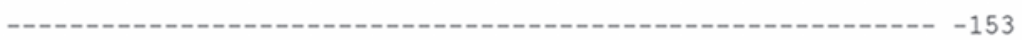
AGGTAGGGCGATCAGTTAGCCCTGAACCCATTTATGCTACAATTGATGATCTCGGCGGA - 354 ---TTCCCTTTGAAAAGGCATGATAAAGTTGATGATCTCAGTAAGGTAGGGCGATCAGT -209 CCTTTCCCTTTGAAAAGGCATGATAAAGTTGATGATCTCAGTAAGGTAGGGCGATCAGT -413

TAGCCCTGAACCCATTTATGCTACAATTGATGATCTCGGCGGACCCTTCCCTTTG $\quad-264$ TAGCCCTGAACCCATTTATGCTACAATTGATGATCTCGGCGGACCTTTCCCTTTG $\quad-468$

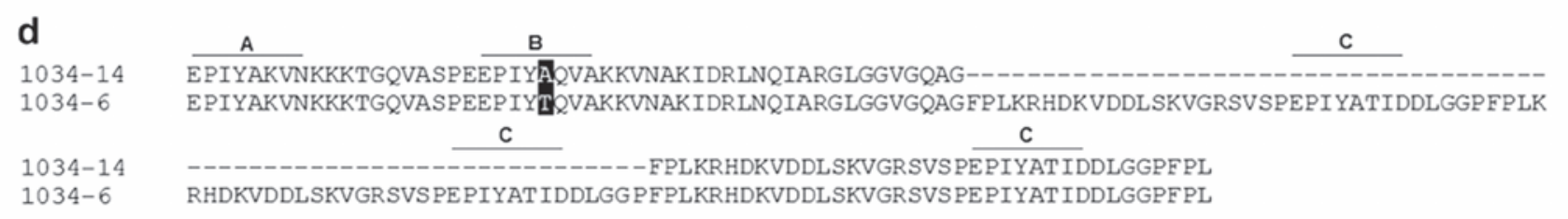

Figure 1. Paired $H$. pylori isolates from a single patient that differ in the cagA 3 ' region. a) Polymerase chain reaction showing different cagA allele size of the isolate 1034-14 and 1034-6. b) Identical RAPD-PCR profiles of the isolates 1034-14 and 1034-6 with two sets of primers, 1281 and 1254. c) The sequencing of the PCR products demonstrated an insertion of 204-bp in the isolate 1034-6 compared with the isolate 1034-14. d) Translation of the nucleotide sequences identified an insertion that corresponds to two identical multimerization sites and EPYIA-C motifs in the 1034-6 isolate. 
marked, resulting in almost complete loss of the oxyntic mucosa (Figure 3c).

\section{Classification and grading of gastri- tis by the Sydney system}

The results regarding the classification and grading of the gastritis according to the Sydney system are shown in Table 1. Only few sparsely distributed MN cells were observed in the antral mucosa of the control animals either 45 days or six months after the inoculation, which was considered the expected cellularity pattern of the antral lamina propria of gerbil in the age ranges studied.

Forty-five days post-inoculation, all animals from the CagA1Ep and CagA3Ep groups exhibited gastric inflammatory infiltrate composed by lymphocytes, macrophages, plasmocytes, and neutrophils, predominantly in the basal mucosa (Figure 4a). These cells were also found in the middle third of the gastric mucosa and in the glandular epithelium in some animals from the CagA3Ep group (Figure 4b). The inflammation was mild in the stomach of most animals of the CagA1Ep group, whereas, moderate degree of gastric inflammation was observed in five gerbils of the CagA3Ep group. A higher degree of gastritis activity $(\mathrm{P}=0.03)$ and a tendency of higher degree of $\mathrm{MN}$ cells $(\mathrm{P}=0.1)$ were observed in the CagA3Ep group when compared with the CagAlEp group (Table 1). Significant differences were observed in respect to all parameters when the controls were compared with the CagA3Ep animals in relation to the scores of MN and PMN cells $(\mathrm{P}<0.001)$ and with the CagA1Ep animals regarding to $\mathrm{MN} \quad(\mathrm{P}<0.001)$ and $\mathrm{PMN}$ $(\mathrm{P}=0.003)$ cells (Table 1$)$.

Six months post-inoculation, the degree of gastric mucosa $\mathrm{MN}(\mathrm{P}=0.01)$ and $\mathrm{PMN}$
$(\mathrm{P}=0.04)$ cells was more pronounced in the animals at six months of infection than in the 45 days post-infection animals (Figure $4 \mathrm{c}-\mathrm{f}$, Table 1). The inflammatory infiltrate was more intense at the base, but expanding to the middle third of the mucosa in most animals, especially in those from the CagA3Ep group. The inflammatory infiltrate also reached the apical third of the mucosa in animals from the CagA3Ep group (Figure 4 e,f). There was a significant higher degree of neutrophils $(\mathrm{P}=0.05)$ in the gastric mucosa of the CagA3Ep when compared to CagA1Ep group. There was also $a$ trend towards an increase score of MN cells in the CagA3Ep group compared with the CagA1Ep group $(\mathrm{P}=0.06)$ (Table 1$)$. The lymphoid follicles were abundant in both groups of infected animals, with no difference between them. Other lesions such as atrophy, intestinal metaplasia and dysplasia were also observed in

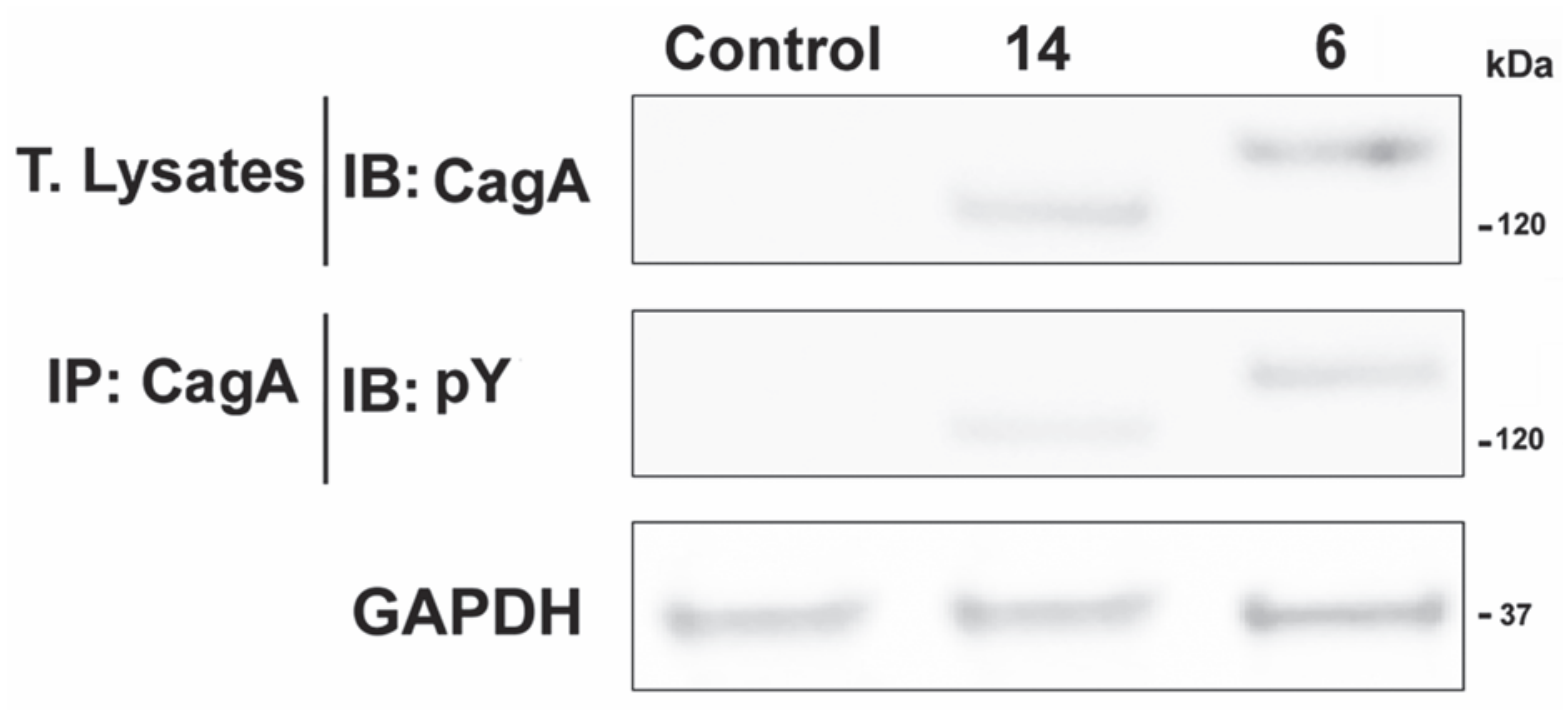

Figure 2. Immunoblotting analysis of CagA protein and tyrosine phosphorylation of $H$. pylori isolates with CagA possessing one or three EPIYA-C segments in human gastric epithelial cells (AGS). Total lysates of AGS cells after co-incubation with $H$. pylori harbouring CagA with one or three EPIYA-C segments (MOI: 100; for 6 h) were immunoblotted (IB) with anti-CagA (A-10) monoclonal antibody. To assess tyrosine phosphorylation, the total lysates were immunoprecipitated (IP) with anti-CagA antibody and then immunoblotted (IB) with the anti-phosphotyrosine monoclonal antibody (PY99). Uninfected AGS cells were used as control. The GAPDH blot served as loading control in each sample.

Table 2. Lesions in the gastric mucosa of Mongolian gerbils of the control group and the groups with six months of infection with Helicobacter pylori.

\begin{tabular}{|c|c|c|c|c|c|c|c|c|c|c|c|c|}
\hline \multirow[t]{2}{*}{ Groups } & \multicolumn{4}{|c|}{ Intestinal metaplasia } & \multicolumn{4}{|c|}{ Atrophy } & \multicolumn{4}{|c|}{ Displasia } \\
\hline & 0 & + & ++ & +++ & 0 & + & ++ & +++ & 0 & + & ++ & +++ \\
\hline CTRL & 8 & 0 & 0 & 0 & 8 & 0 & 0 & 0 & 8 & 0 & 0 & 0 \\
\hline CagAlEp & 6 & 2 & 0 & 0 & 4 & 3 & 1 & 0 & 6 & 2 & 0 & 0 \\
\hline CagA3Ep & 4 & 4 & 0 & 0 & 1 & 4 & 3 & 0 & 5 & 3 & 0 & 0 \\
\hline
\end{tabular}

CTRL, control group consisting of uninfected animal; CagAlEp, CagA-positive with 1 EPIYA-C group; CagA3Ep, CagA-positive with 3 EPIYA-C group; 0, absent; +, mild; ++ moderate; +++, severe Significant differences were observed between the control group and the CagA3Ep group with respect to atrophy $(\mathrm{P}<0.001)$ and intestinal metaplasia $(\mathrm{P}<0.03)$ and a trend toward dysplasia $(\mathrm{P}=0.06)$. The degree of atrophy was also significantly higher $(\mathrm{P}=0.03)$ in the group of animals infected by the strain containing one EPIYA-C motif when compared with the controls. Data were analysed by the two tailed Mann-Whitney U test. 
the infected animals (Figure 5), but not in the control group. Significant differences were observed between the control group and the CagA3Ep group with respect to atrophy $(\mathrm{p}<0.001)$, intestinal metaplasia $(\mathrm{P}<0.03)$ and a trend toward dysplasia $(\mathrm{P}=0.06)$. The degree of atrophy was also significantly higher $(\mathrm{P}=0.03)$ in the group of animals infected by the strain containing one EPIYA-C motif when compared with the controls. The scores of atrophy were higher $(\mathrm{P}=0.05)$ in the CagA3Ep than in the CagAlEp group (Table 2).

a

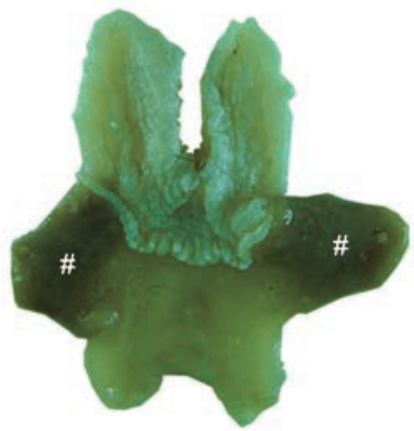

b
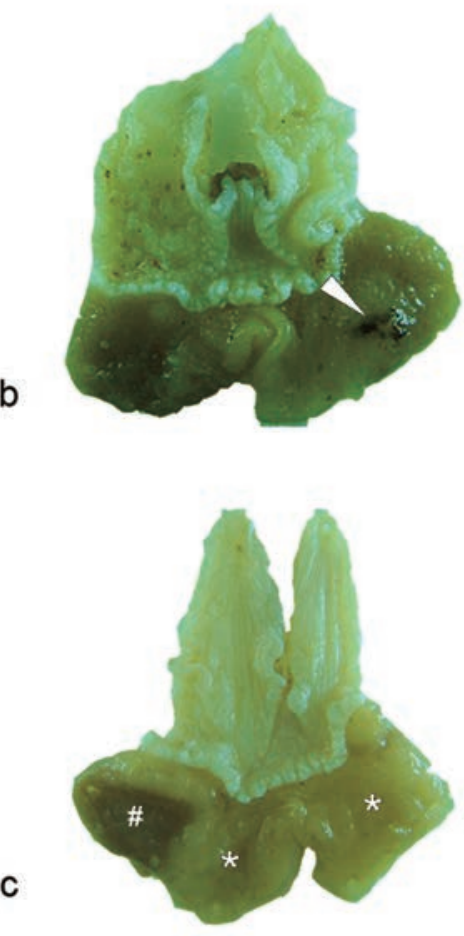

Figure 3. Stomach of Mongolian gerbils infected with $H$. pylori 6 months after the inoculation. a) Normal gastric mucosa of an animal from the control group. b) a flat area with a haemorrhagic aspect (arrowhead) in the antral mucosa of an animal from the CagA1Ep group. c) Expansion of the antral mucosa $(*)$ on the oxyntic mucosa (\#) of an animal from the CagA3Ep group. Scale bars: $2 \mathrm{~cm}$.

\section{Morphometric analysis}

of the inflammatory infiltrate

Forty-five days post-infection, there was a significant increase in the number of inflammatory cells when compared both infected groups with the non-infected animals $(\mathrm{P} \leq 0.003)$. The number of inflammatory cells was significantly higher in the CagA3Ep than in the CagAlEp group, $(\mathrm{P}=0.02)$ (Figure 6a). Confirming these results, the area of the lamina propria occupied by the inflammatory infil- trate after the same period of infection was also significantly larger in both infected groups than in the control group $(\mathrm{P} \leq 0.003)$. CagA3Ep animals showed a larger area than the CagA1Ep animals $(\mathrm{P}=0.01)$ (Figure $6 \mathrm{~b}$ ). Six months post-infection, there were significantly higher number of inflammatory cells and larger area of the antral lamina propria in both infected groups compared with the CTRL group ( $\mathrm{P} \leq 0.004)$. Additionally, there was a statistically significant higher inflammatory infiltrate $(\mathrm{P}=0.01)$ and a larger area of lamina pro-
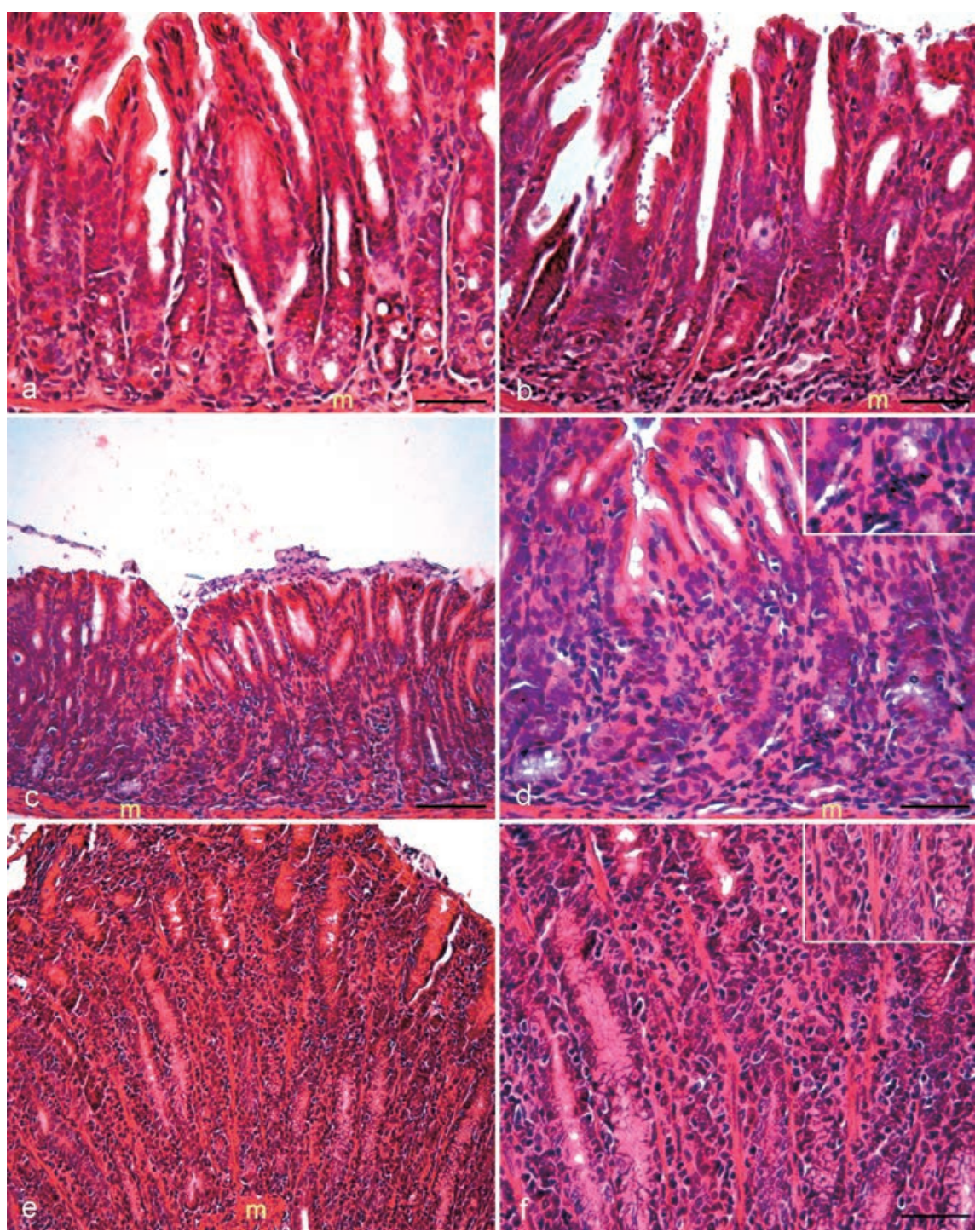

Figure 4. Antral mucosa of Mongolian gerbils infected with Helicobacter pylori: muscularis mucosae (m). a) Antral mucosa of an animal from the CagA1Ep 45 days after the infection; discreet inflammation observed on the basal third of the gastric mucosa. b) Antral mucosa of an animal from the CagA3Ep group 45 days after the infection; inflammation was more severe, also affecting the middle third. c) Antral mucosa of an animal from the CagA1Ep group 6 months after the inoculation. d) Higher magnification of the previous image; insert: detail of the inflammatory infiltrate. e) Antral mucosa of an animal from the CagA3Ep group 6 months after the infection; severe inflammation was observed with increase in the area of the lamina propria. $f$ ) Magnification of panel e); insert: detail of the inflammatory infiltrate; H\&E. Scale bars: a,b,d,f), $50 \mu \mathrm{m}$; c,e), $100 \mu \mathrm{m}$. 
pria $(\mathrm{P}=0.03)$ in the CagA3Ep than in the CagAlEp group (Figure $6 \mathrm{c}$,d). A higher number of cells $(\mathrm{Pp}=0.007)$ and a larger area of lamina propria $(\mathrm{P}=0.05)$ were also observed in the gastric mucosa of infected animals at six months post-infection when compared with the gastric mucosa of animals at 45 days postinfection (Figure 6 e,f).

\section{Discussion}

There are studies demonstrating that infection with CagA-positive H. pylori strains containing more EPYIA-C phosphorylation sites is associated with more severe gastritis, pre-cancerous gastric lesions and gastric cancer.,5,,9,21 However, the associations observed in human beings have not been confirmed experimentally in animal models. The most important finding of this study was the presence of more severe lesions in the gastric mucosa of Mongolian gerbils experimentally infected with $H$. pylori strain carrying three EPIYA-C motifs in the CagA protein and a functional cagPAI. There are several studies in the literature that have evaluated both humans and experimental animals infected by $H$. pylori strains containing or lacking the Cag PAI gene in different parts of the world. However, to date, this is the only study investigating $H$. pylori infection of the gastric mucosa with $H$. pylori strains containing different CagA phosphorylation sites in a classic experimental model for studying this type of infection. It is worthy noticing that CagA from both isolates were translocated and tyrosine phosphorylated in AGS cells and that the results we observed did not depend on either the bacterium load or on great differences between the strains others than the EPIYA-C motif number. In fact, the strains, isolated from a single patient, are frequently clonal. ${ }^{11,26}$ In agreement, all sequenced genes were $\sim 100 \%$ identical when the isolates were compared. Furthermore, both isolates have the same genotypes of $H$. pylori virulence genes involved in gastric inflammation. ${ }^{27}$ Therefore, the difference in the number of EPIYA-C segments of the strains results from intragenomic rearrangement and not from horizontal acquisition from another $H$. pylori strain that would differ in nucleotide sequence by $\sim 6 \%$ on average..$^{28}$ One advantage of this study was to use for inoculation isolates with few passages, which colonize the gastric mucosa of gerbil better than manipulated strains or collection strains. The histopathological results evaluated by the semi-quantitative Sydney system and the quantitative morphometric methods were in accordance; however, the last approach allowed a better discrimination between strains containing one or three EPIYA-C segments in the CagA protein with respect to the number of the inflammatory cells. When crossing the gastric mucous layer, H. pylori adheres to epithelial cells, releases toxins, and triggers an inflammatory process characterised as gastritis, which starts in the antral mucosa. This inflammatory process leads to the accumulation of a superficial acute inflammatory infiltrate composed of lymphocytes, monocytes, plasmocytes, and neutrophils which causes oedema in the lamina propria. Forty-five days after the inoculation, a predominantly antral inflammatory infiltrate, an increase in the area of the lamina propria and the presence of lymphoid follicles that are characteristic markers of the gastritis caused by $H$. pylori ${ }^{3}$ were observed in the infected animals, especially in those carrying
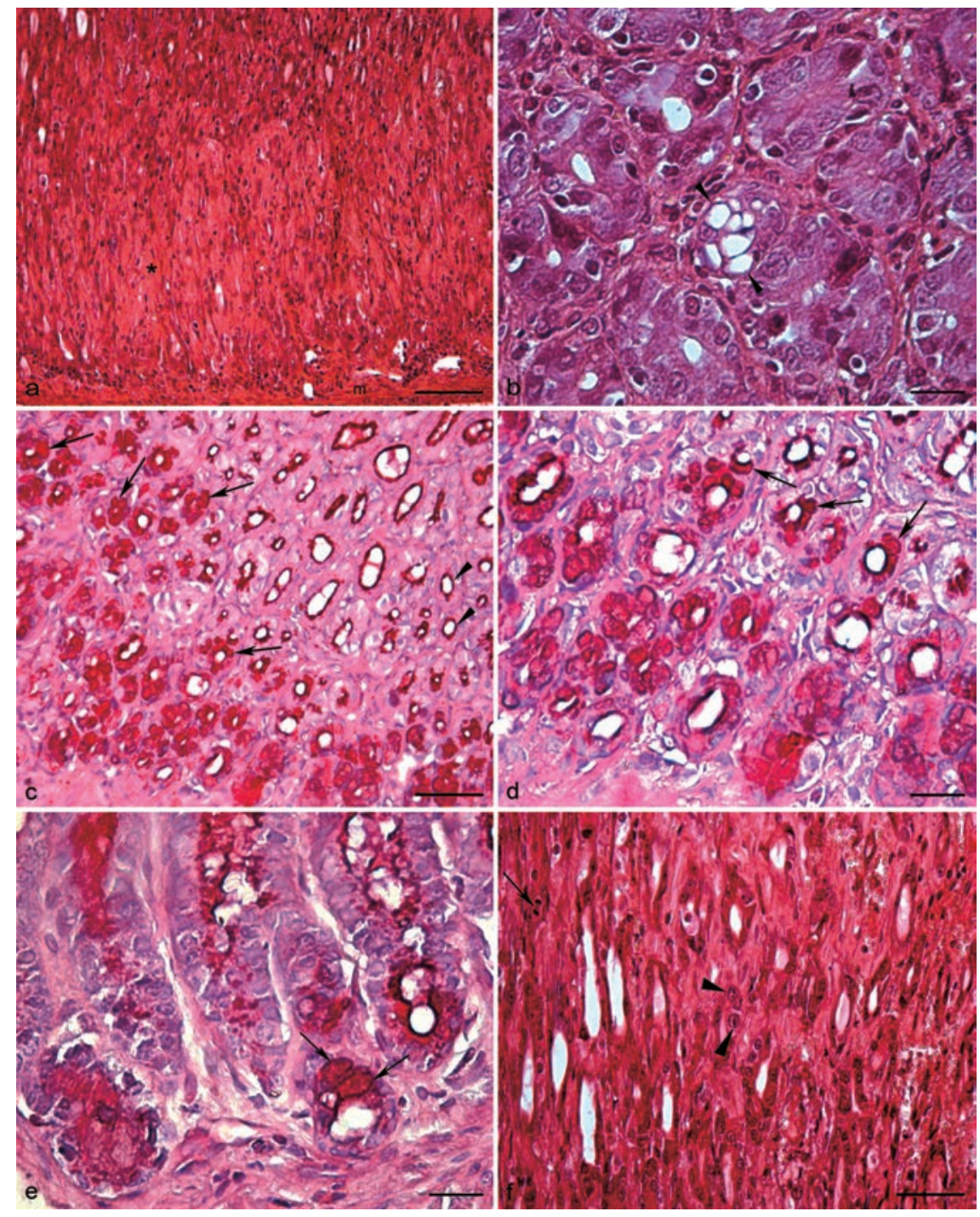

Figure 5. Gastric mucosa of Mongolian gerbils infected with $H$. pylori for six months: muscularis mucosae (m). a) Moderate atrophy of the gastric oxyntic mucosa with the gastric glands replaced by connective tissue in an animal from the CagA3Ep group $\left({ }^{*}\right) ; \mathrm{H} \& \mathrm{E}$; scale bar: $100 \mu \mathrm{m}$. b) Mild intestinal metaplasia in the oxyntic mucosa of an animal from the CagA3Ep group; note the presence of goblet cells in the mucosa (arrowheads); H\&E; scale bar: $20 \mu \mathrm{m}$. c) Gastric mucosa of an animal from the CagA3Ep group with usual glandular epithelium (arrowheads) consisting of mucus producing cells and areas of incomplete intestinal metaplasia (arrows) stained by PAS-Alcian Blue; $\mathrm{pH} 2.5$; scale bar: $50 \mu \mathrm{m} . \mathrm{d}$ ) Detail of the previous figure; Goblet cells positive for PAS-Alcian Blue (arrows); scale bar: $20 \mu \mathrm{m}$. e) Gastric mucosa of an animal from CagA3Ep group with tubular glands containing well-formed goblet cells (arrows) stained by PAS-Alcian Blue; scale bar: $20 \mu \mathrm{m}$. f) Presence of mild dysplasia in the gastric oxyntic mucosa with nuclear pleomorphism and evident nucleoli (arrowheads) as well as mitotic figure (arrow) in an animal from the CagA3Ep group; H\&E; scale bar: $50 \mu \mathrm{m}$. 
the isolate with cagA containing three EPYIA-C segments. The follicles were also more abundant in the gastric mucosa of the CagA3Ep animals. Six months after infection, the inflammatory infiltrate was more intense and composed predominantly of mononuclear cells and neutrophils, characterised as active chronic gastritis. The inflammatory infiltrate was more frequent in the antral mucosa and expanded to the oxyntic mucosa. These results are in agreement with other authors, who demonstrated in gerbils infected with $H$. pylori that the intensity of the parasitism and gastritis are initially greater in the antral mucosa, expanding later to the oxyntic mucosa. ${ }^{28}$ In addition, we observed a significant and progressive increase in the number of inflammatory cells in the groups infected with $H$. pylori (CagA1Ep and CagA3Ep), and this increase was most pronounced in the CagA3Ep group, as demonstrated by the quantitative digital morphometric analysis. A direct relationship between the presence of the CagA protein and more severe forms of the gastric diseases caused by $H$. pylori infection is reported by other authors.5,6,7,29 Álvares et al. found in patients infected with different $H$. pylori strains that the inflammation, activity, atrophy, and IM were significantly more severe in patients infected with cagA-positive samples compared with the negative ones. ${ }^{30}$ Moreover, H. pylori strains with higher numbers of EPIYAs exhibit higher levels of CagA phosphorylation and, therefore, are associated with more severe forms of gastric diseases. In our experiment, in addition to increased inflammation, we also observed significant differences in the inflammatory activity between the CagA1Ep and CagA3Ep groups.

Intestinal metaplasia was also often observed in the animals from the CagA3Ep group 6 months after infection, and in some animals, its development was concomitant with the development of atrophy and dysplasia. This type of lesion is a characteristic finding of chronic atrophic gastritis in the human stomach and is considered the most important risk factor for the development of intestinal carcinoma. The development of intestinal metaplasia has not been observed in any other experimental model of $H$. pylori infection. In the gastric mucosa of gerbils infected with $H$. pylori strains $\mathrm{CagA}^{+}$, gastric mucosa lesions are reportedly more severe, including chronic atrophic gastritis, incomplete-type IM and/or an increased risk of gastric cancer compared with animals infected with CagA-negative $H$. pylori strains or with a mutant form of CagA. ${ }^{29,31}$ Thus, the gerbil was an ideal model to investigate the histogenesis of inflammation and metaplasia and its relationship with carcinogenesis. The morphometric analysis allowed us to quantify the level of inflamma- tion, providing information that was impossible to obtain through semi-quantitative analysis. The inflammation induced by $H$. pylori infection is a chronic active gastritis, characterised by lymphocytic and neutrophilic infiltration in the gastric mucosa. From the inflammatory infiltrate, foci of intestinal metaplasia with subsequent atrophy of the mucosa, aggregates, and lymphoid follicles occur concurrently, and the foveolar epithelium is replaced by a regenerative epithelium with reduced mucin secretion. ${ }^{21}$ In the present study, the atrophy
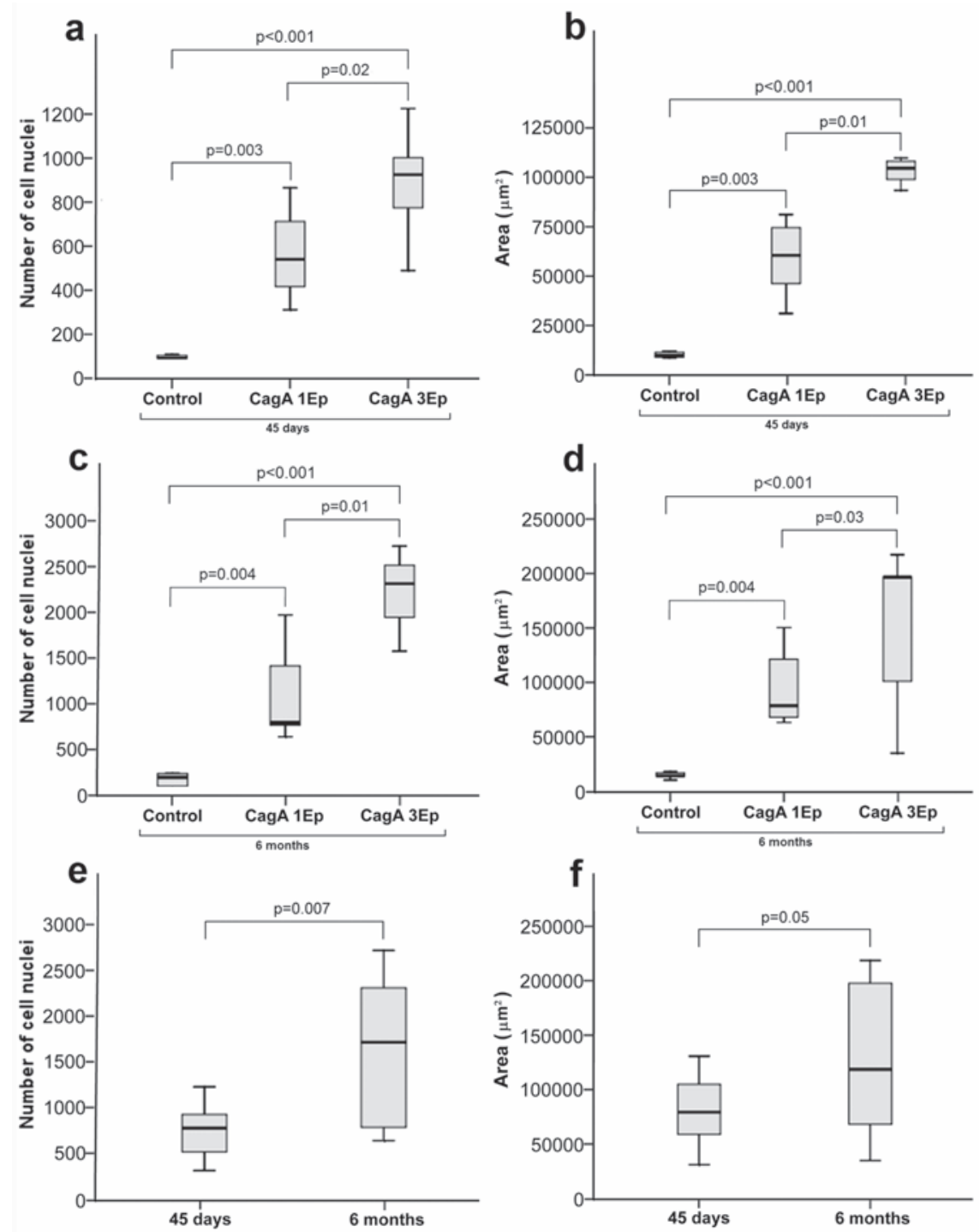

Figure 6. Box plots representing the total number of inflammatory cells and the lamina propria area in 15 fields of the gastric mucosa of Mongolian gerbils infected with $\mathrm{H}$. pylori strains containing one or three CagA EPIYA-C phosphorylation $C$ sites and uninfected animals as a control group at 45 days $(\mathrm{a}, \mathrm{b})$ or six months $(\mathrm{c}, \mathrm{d})$ after the inoculation; each group is composed by $\mathbf{8}$ animals. e, f) Represent the higher number of inflammatory cells and increased lamina propria area respectively in infected animals with six months of infection compared with those with 45 days of infection; the upper and lower limits of the boxes represent the $75^{\text {th }}$ and $25^{\text {th }}$ percentiles, respectively; the horizontal bar across the box indicates the median and capped bars indicate the minimum and maximum data values. Data were analyzed by the two tailed Mann-Whitney $U$ Test. 
tion of the signal peptide that modulates the growth and differentiation of the gastric progenitor cells, resulting in an increased proliferation of undifferentiated cells. On the other hand, the atrophy induces a hypochlorhydria condition in the gastric mucosa, with consequent stimulation of gastrin-producing cells, inducing hypergastrinemia, which also results in increased proliferation of gastric mucosal cells. ${ }^{34}$ In fact, some studies reported a higher frequency of malignant gastric lesions in individuals over 50 years of age with chronic atrophic gastritis. ${ }^{35}$ Thus, for several authors, atrophic gastritis is more important than intestinal metaplasia as a risk factor for the development of gastric cancer. ${ }^{36}$ Like atrophy, dysplasia occurred 6 months after infection. This lesion was characterised by an increase in the proliferation, loss of cell differentiation, and cellular atypia, mainly karyomegaly. We properly demonstrated the entire sequence of the lesions in the gastric mucosa caused by $H$. pylori in an experimental model that is most similar to human infection. However, to assess the effects of different $H$. pylori strains on the likelihood of developing gastric carcinoma, an evaluation of the infection for periods longer than 6 month is required.

Our results may be explained by the higher SHP-2 activity induced by the isolate with three EPIYA-C phosphorylation sites. It has been demonstrated that phosphorylated CagA protein forms a physical complex with SHP-2 phosphatase that activates MAPK/ERK 1/2 signaling pathway. ERK regulates inflammation by stimulating neutrophil adhesion and superoxide generation, and mediates pro-inflammatory cytokine secretion. ${ }^{37}$ Furthermore, ERK leads to activation of genes that encode antiapoptotic proteins, deregulation of the cell growth and differentiation with an increase of the epithelial cell turnover, which enhance the risk of damaged cells acquire pre-cancerous genetic changes. Thus, $H$. pylori strain carrying EPIYA with multiple $\mathrm{C}$ repeats leads to more SHP-2 activity and consequently higher degree of gastric inflammation, atrophic gastritis, gastric metaplasia and increased risk of gastric cancer. ${ }^{6,38}$

Concluding, we reproduced in Mongolian gerbil the pattern of gastric lesions observed in human beings infected with $H$. pylori strain with a greater number of EPIYA-C segments in the CagA protein. The animals developed gastric histopathological lesions that followed the steps of the carcinogenesis model proposed by Pelayo-Correa for intestinal gastric carcinoma, including chronic gastritis, atrophy, intestinal metaplasia and dysplasia. ${ }^{31}$

\section{References}

1. Pounder RE, Ng D. The prevalence of Helicobacter pylori infection in different countries. Aliment Pharm Therap 1995;9: 33-9.

2. Blaser MJ. Helicobacter pylori and gastric diseases. BMJ 1998;316:1507-10.

3. Dixon MF, Genta RM, Yardley JH, Correa P. Classification and grading of gastritis. The updated Sydney System. Am J Surg Pathol 1996;10:1161-181.

4. Backert S, Schwarz T, Miehlke S, Kirsch C, Sommer C, Kwok T, et al. Functional analysis of the cag pathogenicity island in Helicobacter pylori isolates from patients with gastritis, peptic ulcer, and gastric cancer. Infect Immun 2004;2:1043-56.

5. Backert S, Selbach M. Role of type IV secretion in Helicobacter pylori pathogenesis. Cellular Microbiology 2008;8:1573-81.

6. Batista SA, Rocha AG, Rocha AMC, Saraiva IE, Cabral MM, Oliveira RC, et al. Higher number of Helicobacter pylori CagA EPIYA C phosphorylation sites increases the risk of gastric cancer, but not duodenal ulcer. BMC Microbiology 2011;61:1-7.

7. Hatakeyama M. Oncogenic mechanisms of the Helicobacter pylori CagA protein. Nat Rev Cancer 2004;4:688-94.

8. Chuang CH, Yang HB, Sheu SM, Hung KH, Wu JJ, Cheng HC, et al. Helicobacter pylori with stronger intensity of CagA phosphorylation lead to an increased risk of gastric intestinal metaplasia and cancer. BMC Microbiology 2011;121:1-7.

9. Queiroz DMM, Mendes EN, Rocha GA. Indicator Medium for Isolation of Campylobacter pylori. J Clin Microbiol. 1987;12:2378-79.

10. Yamaoka Y, Kodama T, Kashima K, Graham DY, Sepulveda AR. Variants of the 3 ' region of the cagA gene in Helicobacter pylori isolates from patients with different H. pylori-associated diseases. J Clin Microbiol 1998;8:2258-63.

11. Suerbaum S, Smith JM, Bapumia K, Morelli G, Smith NH, Kunstmann E, et al. Free recombination within Helicobacter pylori. Proc Natl Acad Sci 1998; 95:12619-24.

12. Akopyanz N, Bukanov NO, Westblom TU, Kresovich S, Berg DE. DNA diversity among clinical isolates of Helicobacter pylori detected by PCR-based RAPD fingerprinting. Nucleic Acids Res 1992;19:5137-42.

13. Achtman M, Azuma T, Berg DE, Ito Y, Morelli G, Pan ZJ, et al. Recombination and clonal groupings within Helicobacter pylori from different geographical regions. Mol Microbiol 1999;32:459-470.

14. Kauser F, Hussain MA, Ahmed I, Ahmad N, Habeeb A, Khan AA, et al. Comparing genomes of Helicobacter pylori strains from the high-altitude desert of Ladakh, India. J Clin Microbiol 2005;43:1538-45.

15. Oliveira AG, Santos A, Guerra JB, Rocha, GA, Rocha AMC, Oliveira CA, et al. babA2and cagA-positive Helicobacter pylori strains are associated with duodenal ulcer and gastric carcinoma in Brazil. J Clin Microbiol 2003;4:3964-66.

16. Moura SB, Costa RFA, Anacleto C, Rocha GA, Rocha AMC Queiroz DMM. Single nucleotide polymorphisms of Helicobacter pylori dupA that lead to premature stop codons. Helicobacter 2011;17:176-80.

17. Azuma T, Yamakawa A, Yamazaki S, Ohtani M, Ito Y, Muramatsu A, et al. Distinct Diversity of the cag Pathogenicity Island among Helicobacter pylori Strains in Japan. J Clin Microbiol 2004;42(6): 2508-17.

18. Ashour AAR, Collares GB, Mendes EN, Gusmão VR, Queiroz DMM, Magalhães PP, et al. iceA genotypes of Helicobacter pylori strains isolated from Brazilian children and adults. J Clin Microbiol 2001;39: 1746-50.

19. Liang XH, Zhang YN, Wang YJ, Kang XX. Analysis of translocation of the CagA protein and induction of a scattering phenotype in AGS cells infected with Helicobacter pylori. Biomed Environ Sci 2009;22:394-400.

20. Monteiro L, Gras N, Vidal R, Cabrita J , Mégraud F. Detection of Helicobacter pylori DNA in human feces by PCR: DNA stability and removal of inhibitors. J Microbiol Methods 2001;45:89-94.

21. Clayton CL, Kleanthous H, Coates PJ, Morgan DD, Tabaqchali S. Sensitive detection of Helicobacter pylori by using polymerase chain-reaction. J Clin Microbiol 1992;30:192-00.

22. Peek RM Jr, Miller GG, Tham KT, PerezPerez GI, CoverT, Atherton JC, et al. Detection of Helicobacter pylori gene expression in human gastric mucosa. J Clin Microbiol 1995;33:28-32.

23. Rocha GA, Queiroz DMM, Mendes EN, Lage AP, Barbosa AJA. Simple carbolfuchsin staining for showing Campylobacter pylori and other spiral bacteria in gastric mucosa. J Clin Pathol 1989; 42:1004-05.

24. Wirth HP, Beins MH, Yang M, Tham KT , Blaser MJ. Experimental infection of Mongolian gerbils with wild-type and Helicobacter pylori strains. Infect Immun 1998; 66:4856-66.

25. Pacheco CM, Queiroz-Junior CM, Maltos KL, Caliari MV, Pacheco DF, Duarte ID, et al. Crucial role of peripheral kappa-opioid receptors in a model of periodontal disease in rats. J Periodontal Res 2008; 6:730-36. 
26. Alm RA, Ling LS, Moir DT, King BL, Brown ED, Doig PC, et al. Genomic-sequence comparison of two unrelated isolates of the human gastric pathogen Helicobacter pylori. Nature1999; 397:176-80.

27. Kalali B, Mejías-Luque R, Javaheri A, Gerhard M. H. pylori virulence factors: influence on immune system and pathology. Med Inflamm 2014;ID 426309, 9 pages.

28. Aras RA, Lee Y, Kim SK, Israel D, Peek RM, Blaser MJ. Natural variation in populations of persistently colonizing bacteria affects human host cell phenotype. J Infect Dis 2003;188:486-96.

29. Ohnita K, Isomoto H, Honda S, Wada A, Wen CY, Nishi Y, et al. Helicobacter pylori strain-specific modulation of gastric inflammation in Mongolian gerbils. World J Gastroenterol 2005;10:1549-53.

30. Álvares MMD, Marino M, Oliveira CA, Mendes CC, Costa ACF, Guerra J, et al.
Características da gastrite crônica associada a Helicobacter pylori: aspectos topográficos, doenças associadas e correlação com 0 status cagA. J Bras Patol Med Lab 2006;1:51-9.

31. Wiedemann T, Loell E, Stoeckelhuber M, Stolte M, Haas R, Rieder G. Helicobacter Pylori cag-Pathogenicity Island-Dependent Early Immunological Response Triggers Later Precancerous Gastric Changes in Mongolian Gerbils. PLoS ONE 2009;4:1-13.

32. Murakami M, Fukuzawa M, Yamamoto M, Hamaya K, Tamura Y, Sugiyama A, et al. Effects of Helicobacter pylori infection on gastric parietal cells and E-cadherin in Mongolian gerbils. J Pharmacol Sci 2013;121: 305-11.

33. Neu B, Puschmann AJ, Mayerhofer A, Hutzler P, Grossmann J. TNF-alpha induces apoptosis of parietal cells. Biochem Pharmacol 2003;65:1755-60.
34. Fox JG, Wang TC, Rogers AB, Poutahidis T, Ge Z. Host and microbial constituents influence Helicobacter pylori-induced cancer in a murine model of hypergastrinemia. Gastroenterol 2003;124:1879-90.

35. Vannella L, Lahner E, Osborn J, Bordi C, Miglione M, Delle FG, et al. Risk factors for progression to gastric neoplastic lesions in patients with atrophic gastritis. Aliment Pharmacol Ther 2010;31:1042-50.

36. Fox JG, Wang TC. Inflammation, atrophy, and gastric cancer. J Clin Invest 2007; 117: $60-9$.

37. Higashi H, Tsutsumi R, Muto S, Sugiyama T, AzumaT, Asaka M, et al. SHP-2 tyrosine phosphatase as an intracellular target of Helicobacter pylori CagA protein. Science 2002;295:683-6.

38. Correa P, Piazuelo MB. The gastric precancerous cascade. J Dig Dis 2012; 13:2-9. 